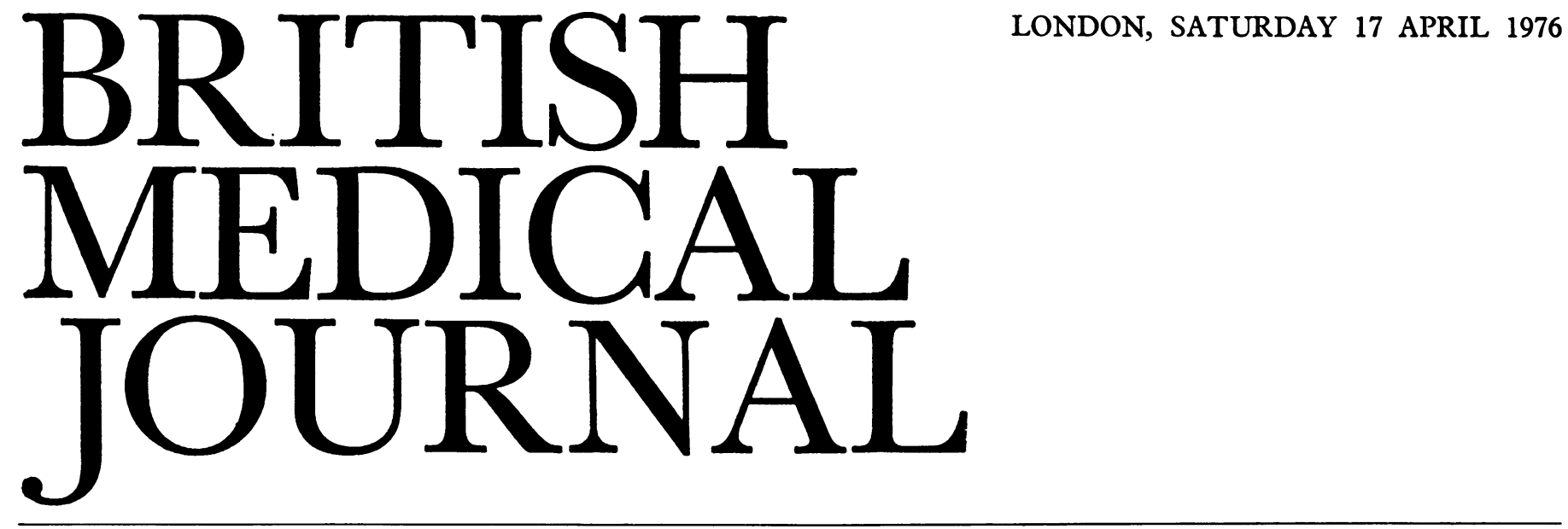

\title{
Vaccination against meningitis
}

The prevalence of meningococcal meningitis has increased worldwide over the last ten years: it now gives rise to concern in many countries where previously it was not considered to be much of a public health problem. Major epidemics have occurred in a few countries and there have been local outbreaks as close to hand as Devon ${ }^{1}$ and Dublin. ${ }^{2}$ The number of cases notified ${ }^{4}$ in Britain trebled between 1967 and 1974 but then decreased from 1296 in 1974 to 840 in 1975 . During the first four weeks of January 1976 there were 106 notified cases and 12 deaths assigned to meningococcal meningitis in England and Wales. ${ }^{3}$

The fulminating form of meningococcal disease usually results in death within 4 to 48 hours of onset, and the acute septicaemic form follows an almost equally severe course. Meningococcal meningitis, however, has a lower fatality rate of about $50 \%$ which can be reduced to around $10 \%$ by early diagnosis and parenteral administration of penicillin. All strains of Neisseria meningitidis isolated from cerebrospinal fluid or blood before 1963 were regarded as sensitive to sulphadiazine, but since then the incidence of resistant isolates has increased to $11 \%$ in Britain $^{3}$ and is probably much higher in the United States. ${ }^{5}$

Meningococcal infection (as opposed to disease) commonly occurs during childhood and adolescence, but ordinarily it leads to no more than an asymptomatic nasopharyngeal state which persists for several weeks or months. This accounts for the nasopharyngeal carrier rate of $2-5 \%$ in cross sections of the population at any one time with the highest rates in children and young adults. ${ }^{6}$ Cases of meningococcal disease occur sporadically, mainly during the winter months and in children living under crowded or poor socioeconomic conditions or in young recruits at military or naval barracks. The incidence of cases varies from time to time and may reach epidemic proportions. Whenever there is an increase in incidence there is a substantial rise in the number of healthy carriers in the community.

Most cases of meningococcal meningitis are attributable to serogroup $\mathrm{A}, \mathrm{B}$, or $\mathrm{C}$ strains of $N$ meningitidis, but infection and occasionally disease can also be due to other groups (D, X, Y, Z, 29-E, W135) or to ungroupable strains. ${ }^{7}$ W'hereas group A strains were apparently responsible for most cases of meningococcal meningitis in the United States before 1963 they were succeeded by group B strains from 1964 to 1967 and subsequently by group C strains. ${ }^{8}$ In Britain, $60 \%$ of cases of meningococcal meningitis in 1974 and 1975 were due to group
B strains, $15-20 \%$ to group C, and about $10 \%$ to group A. ${ }^{3}$ The 1971 epidemic in Brazil was predominantly due to serogroup C strains, ${ }^{9}$ while epidemics in the Sudan, ${ }^{10}$ Egypt, ${ }^{11}$ Finland, ${ }^{12}$ and Brazil (in 1974) ${ }^{13}$ were predominantly due to group A.

A single attack of meningococcal disease confers lasting immunity against further attacks, indicating that it stimulates resistance to all strains of $N$ meningitidis, irrespective of group. Similarly, an asymptomatic infection with prolonged nasopharyngeal carriage will initiate, reinforce, and extend immunity to all strains. ${ }^{71415}$ This accounts for the passive protection derived transplacentally by infants ${ }^{16}$ and the high level of acquired immunity in older adults. Acquired immunity may be shown to be related to the presence of bactericidal antibodies in the serum. ${ }^{1718}$

In 1969 Artenstein et al developed a Cetavlon-precipitated, high molecular weight meningococcal polysaccharide vaccine ${ }^{1920}$ which appeared to provide a safe and effective means of protecting children and servicemen against meningococcal disease. ${ }^{21-25}$ Two vaccines, produced from $N$ meningitidis groups $\mathrm{A}$ and $\mathrm{C}$ respectively, were shown to stimulate production of bactericidal antibodies ${ }^{21}$ which persisted for at least 5 years ${ }^{26}$ and were effective in the control of homologous group epidemics. ${ }^{10-12}$ Both are licensed for use in the United States, but nevertheless they are not recommended for routine vaccination of civilians, ${ }^{27}$ for they have serious limitations.

Natural infection with a strain of $N$ meningitidis stimulates the production of broad spectrum bactericidal antibodies and so gives immunity against the various groups and ungroupable strains; but the group $\mathrm{A}$ and $\mathrm{C}$ polysaccharide vaccines stimulate production of group-specific bactericidal antibodies only, and so give protection only against homologous group infection. Widespread use of either or both vaccines in Britain, for instance, where $60 \%$ of meningococcal disease is attributable to group B strains, would have little effect on its incidence. Even in countries where either group A or C strains predominate immunisation of the susceptible section of the population with these vaccines would leave individuals susceptible to other group or ungroupable strains and, indeed, facilitate their spread. ${ }^{2328}$ Though some of these organisms are considered to be of low pathogenicity their increased incidence could be accompanied by a change in their importance. In the circumstances the polysaccharide vaccines are reserved to quell epidemics or institutional outbreaks primarily due to group A or $\mathrm{C}$ strains. The delay of 5-7 days that occurs before antibodies 
are formed in response to vaccination and the specificity of the response greatly limit the usefulness of vaccines for the protection of close contacts of cases of meningococcal meningitis. High costs of vaccine production and distribution and the instability of group A vaccine at ambient temperatures add further difficulties. Clearly, there is a need and room for further vaccines, as is implicit in the recent call made by a World Health Organisation Study Group for urgent development of effective tools for the control of the disease. ${ }^{29}$

${ }^{1}$ Easton, D M, et al, British Medical fournal, 1974, 1, 507.

2 Cahalane, S F, and Waters, M, Lancet, 1975, 2, 120.

3 British Medical fournal, 1976, 1, 466.

4 Office of Population Censuses and Surveys, Registrar General's Weekly Return for England and Wales, Reference WR 76/4. London, HMSO.

5 Wiggins, G L, Hollis, D G, and Weaver, R, American fournal of Public Health, 1973, 63, 59 .

${ }^{6}$ Fraser, P K, et al, Lancet, 1973, 1, 1235.

7 Artenstein, M S, Schneider, H, and Tingley, M D, Bulletin of the World Health Organisation, 1971, 45, 275.

${ }^{8}$ Surveillance Summary-Meningococcal Disease, Morbidity and Mortality Weekly Report, 1973, 22, 58.

${ }^{9}$ De Morais, J S, et al, Fournal of Infectious Diseases, 1974, 129, 568.

${ }_{10}$ Erwa, H H, et al, Bulletin of the World Health Organisation, 1973, 49, 301.

11 Wahdan, M H, et al, Bulletin of the World Health Organisation, 1973, 48, 667.

12 Mäkelä, P H, et al, Lancet, 1975, 2, 883.

13 Pan American Health Organisation, Morbidity and Mortality Weekly Report, 1974, 23, 349.

14 Goldschneider, I, Gotschlich, E C, and Artenstein, M S, fournal of Experimental Medicine, 1969, 129, 1327.

${ }^{15}$ Reller, L B, MacGregor, R R, and Beaty, H N, fournal of Infectious Diseases, 1973, 127, 56.

${ }^{16}$ Groover, R V, Sutherland, J M, and Landing, B H, New England fournal of Medicine, 1961, 264, 1115.

17 Goldschneider, I, Gotschlich, E C, and Artenstein, M S, Fournal of Experimental Medicine, 1969, 129, 1307.

18 Artenstein, M S, et al, fournal of Infectious Diseases, 1971, 124, 277.

19 Gotschlich, E C, Liu, T Y, and Artenstein, M S, fournal of Experimental Medicine, 1969, 129, 1349.

${ }^{20}$ Gotschlich, E C, Goldschneider, I, and Artenstein, M S, fournal of Experimental Medicine, 1969, 129, 1367.

21 Gotschlich, E C, Goldschneider, I, and Artenstein, M S, fournal of Experimental Medicine, 1969, 129, 1385.

22 Artenstein, M S, et al, fournal of Infectious Diseases, 1970, 121, 372.

23 Artenstein, M S, et al, New England fournal of Medicine, 1970, 282, 417.

24 Gold, R, and Artenstein, M S, Bulletin of the World Health Organisation, $1971,45,279$.

25 Artenstein, M S, et al, Military Medicine, 1974, 139, 91.

${ }^{26}$ Brandt, B L, and Artenstein, M S, Fournal of Infectious Diseases, 1975, 131, S69.

27 Public Health Service, Advisory Committee on Immunisation Practices, Morbidity and Mortality Weekly Report, 1975, 24, 381.

28 Smilack, J D, Annals of Internal Medicine, 1974, 81, 740.

29 World Health Organisation, Press Release WHO 37, 5 November 1975.

\section{Puerperal mastitis}

Breast-feeding seems to be returning to fashion and is being recommended ${ }^{1}$ by the DHSS, yet even so less than half of all new mothers elect to breast-feed..$^{2-4}$ As many as $9 \%$ of these young women may develop puerperal mastitis, ${ }^{5} 6$ and, while some authorities state that weaning is never necessary, ${ }^{7}$ others recommend it. ${ }^{8}$ In all cases, however, the mastitis is painful and distressing. So how can it be prevented?

Sporadic and epidemic forms of puerperal mastitis are recognised. In sporadic mastitis the nipple is fissured and milk stagnates, ${ }^{9}$ representing the end result of poor nursing technique. ${ }^{7}$ Engorgement of the breast prevents the infant drawing both the nipple and the adjacent areola into its mouth: the nipple can only be nibbled and may be damaged, complete drainage of the breast is not achieved, and the scene is set for infection. At first any cellulitis is confined to the interlobular connective tissue, so that it is rare for pus to be expressed from the nipple. ${ }^{10}$ The sequence of events can be prevented by the simple expedient of manually expressing a little milk immediately before nursing to reduce the tension in the breast.? Normal suckling and a strong "let-down" will then complete breast emptying without injury to the nipple.

Epidemic mastitis is a primary duct infection caused by highly virulent strains of Staphylococcus aureus, often now penicillin-resistant. ${ }^{910}$ Pus can always be expressed from the nipple and organisms cultured from the milk. Epidemiological studies have shown that the newborn baby's nasopharynx is the reservoir from which infection is transferred to the mother. ${ }^{11} 12$ The chain of infection is nursery-infant-mother and is initiated by cross-infection, indicating poor nursery asepsis. In an epidemic reported by Ravenholt et al ${ }^{13}$ staphylococcal disease was completely eliminated when temporary closure of the nursery "removed the handle from the pump.'

What is the correct management of a mother who develops puerperal mastitis? The principles governing treatment, clearly defined by Taylor and Way, ${ }^{14}$ are emptying of the breast and control of infection. The breast can be emptied either by suckling or manual expression. There is no place for "resting the inflamed part," as this simply encourages engorgement. Applebaum ${ }^{7}$ advises more frequent nursing in the early stages of the disease, a view supported by numerous reports. 91415 Despite the presence of staphylococcal pyolactia no illness was recorded in any infant in these series. Even established sporadic mastitis need not interrupt lactation, but it is usually advisable to stop nursing with the epidemic form, ${ }^{9}$ since otherwise there is likely to be continued reinfection from the infant's nasopharynx.

Abscess formation occurs in $5-11 \%$ of patients with mastitis $^{915}$ and is a definite contraindication to suckling, though feeding may continue at the contralateral breast. The potential dangers of attempting to suppress lactation with stilboestrol prevent its use in mastitis, ${ }^{16}$ and in any case continued lactation produces speedier resolution. Indeed, inhibition of lactation by stilboestrol is associated with a higher incidence of recurrent abscess ${ }^{17}$; but bromocriptine may prove to be the drug of choice when lactation has to be suppressed. ${ }^{18}$ Encouraging reports from China on the value of extracts from the plant genus Gleditschia warrant further investigation. ${ }^{19}$

The second principle, control of infection, is achieved with antibiotics. Penicillin, given early in an attack, used to produce rapid resolution of puerperal mastitis 11 with a reduction in the incidence of breast abscess. ${ }^{20}$ Flucloxacillin has now become the antibiotic of choice since the emergence of penicillin-resistant strains. ${ }^{21}$ The early use of antibiotics is vital: breast abscess is a common sequel in patients left untreated longer than twenty-four hours. ${ }^{15}$ Delay may result in the development of the "non-tender mass simulating a carcinoma," once mistakenly thought by surgeons to represent antibiotic abuse. ${ }^{22}$ If there is an established breast abscess, however, antibiotics simply delay the inevitable need for drainage, best accomplished by the primary suture technique of Goodman and Benson. ${ }^{16}$

An ounce of prevention is worth a pound of cure. ${ }^{7}$ Sporadic mastitis may be prevented by teaching women a good nursing technique, while control of cross-infection will eliminate the epidemic form. When prevention fails the subclinical stage of lactational mastitis may be recognised cytologically and arrested by oxytocin. ${ }^{23}$ Development of the overt disease requires immediate antibiotic therapy, together with continued breast emptying, ideally by suckling. Lactation need not be suppressed except in epidemics resulting from highly 